\title{
Clinical and molecular implications of NAB2-STAT6 fusion variants in solitary fibrous tumour
}

\author{
Tatiana Georgiesh ${ }^{1,2}$, Heidi Maria Namløs ${ }^{2}$, Nitin Sharma ${ }^{2}$, \\ Susanne Lorenz ${ }^{2}$, Ola Myklebost ${ }^{3}$, Bodil BJerkehagen ${ }^{1,4}$, \\ Leonardo A. MeZa-ZePeda ${ }^{2, *}, K_{\text {Jetil }}$ Boye $^{2,5, *}$ \\ ${ }^{1}$ Department of Pathology, Norwegian Radium Hospital, Oslo University Hospital, Oslo, \\ Norway; ${ }^{2}$ Department of Tumour Biology, Institute for Cancer Research, Norwegian Radium \\ Hospital, Oslo University Hospital, Oslo, Norway; ${ }^{3}$ Department of Clinical Science, Uni- \\ versity of Bergen, Bergen, Norway; ${ }^{4}$ Institute of Clinical Medicine and Institute of Oral \\ Biology, University of Oslo, Oslo, Norway; ${ }^{5}$ Department of Oncology, Norwegian Radium \\ Hospital, Oslo University Hospital, Oslo, Norway; " these authors share senior authorship
}

\begin{abstract}
Summary
Solitary fibrous tumour (SFT) is a mesenchymal neoplasm characterised by pathognomonic NAB2-STAT6 gene fusions. The clinical implications and prognostic value of different fusion variants has not been clarified. In the current study, we explore the clinicopathological, prognostic and molecular differences between tumours with different fusions. Thirty-nine patients with localised, extrameningeal SFT were included, of whom 20 developed distant recurrence and 19 were without recurrence after long term follow-up. Capture-based RNA sequencing identified 12 breakpoint variants, which were categorised into two groups based on the STAT6 domain composition in the predicted chimeric proteins. Twenty-one of $34(62 \%)$ sequenced tumours had fusions with most of the STAT6 domains intact and were classified as STAT6-Full. Thirteen tumours $(38 \%)$ contained only the transactivation domain of STAT6 and were classified as STAT6-TAD. Tumours with STAT6-TAD fusions had a higher mitotic count $(p=0.016)$ and were associated with inferior recurrence-free interval $(p=0.004)$ and overall survival $(p=0.012)$. Estimated 10year recurrence-free survival was $25 \%$ for patients with STAT6-TAD tumours compared to $78 \%$ for the STAT6-Full group. Distinct transcriptional signatures between the fusion groups were identified, including higher expression of FGF2 in the STAT6-TAD group and IGF2, EGR2, $P D G F R B, S T A T 6$ and several extracellular matrix genes in STAT6-Full tumours. In summary, we demonstrate that NAB2-STAT6 fusion variants are associated with distinct clinicopathological and molecular characteristics and have prognostic significance in extrameningeal SFT.
\end{abstract}

Key words: Solitary fibrous tumour; fusion variants; NAB2; STAT6; recurrence; prognosis; survival; gene expression.

Received 13 September, accepted 20 November 2020 Available online 19 March 2021

\section{INTRODUCTION}

Solitary fibrous tumour (SFT) is an uncommon mesenchymal neoplasm characterised by a pathognomonic gene fusion between NAB2 (NGFI-A binding protein 2) and STAT6 (signal transducer and activator of transcription 6), which is suggested to be the central molecular alteration driving tumour development and progression. ${ }^{1,2}$ The presence of the fusion has been confirmed in nearly all SFTs ${ }^{3,4}$ and the introduction of STAT6 as a robust immunohistochemical marker has significantly improved diagnostics. ${ }^{5-7}$

NAB2 and STAT6 are neighbour genes localised on the long arm of chromosome 12 and transcribed in opposite directions. In SFT, an intrachromosomal inversion places the genes in the same orientation which results in an in-frame fusion transcribed from the $N A B 2$ promoter. ${ }^{1,2}$ Thereby, the transcriptional repressor $N A B 2$ inherits the transactivation domain (TAD) of STAT6, which turns the chimeric protein into a transcriptional activator of early growth response factor 1 (EGR1) responsive genes. This event is believed to trigger tumour development.

The NAB2 protein is composed of 3 domains: NCD1 (NAB-conserved domain 1), NCD2 (NAB-conserved domain 2) and CID (CHD4-interacting domain), a C-terminal repression domain. ${ }^{8,9}$ The STAT6 protein has five domains: a protein interaction domain (PID), an all-alpha domain, a DNA-binding domain (DBD), a Src homology-2 domain (SH2) and a transactivation domain (TAD). ${ }^{9}$ The domain structure of the proteins is summarised in Fig. 1A.

More than 40 breakpoint variants including different exons of the fusion partners have been reported. Among these, NAB2ex4-STAT6ex $2 / 3$ and NAB2ex6-STAT6ex16/17 are the most frequent. ${ }^{4,10-14}$ Barthelmess and co-workers showed that NAB2ex4-STAT6ex2/3 was associated with fewer recurrences, pleuropulmonary localisation, larger tumour size, lower mitotic count and older age at presentation compared to NAB2ex6-STAT6ex16/17. ${ }^{13}$ Several 
A

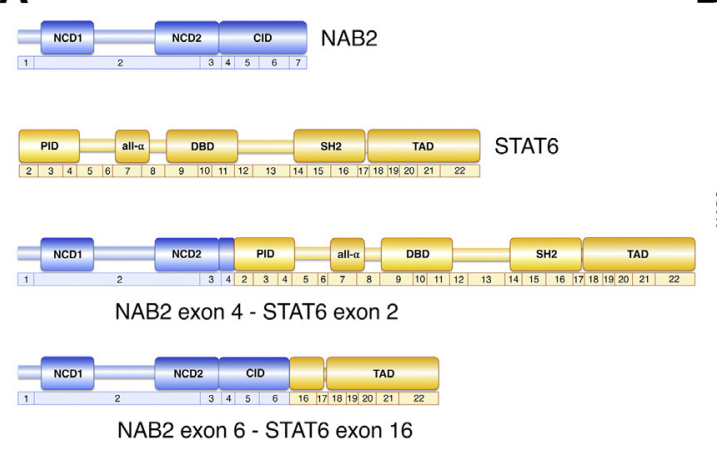

B

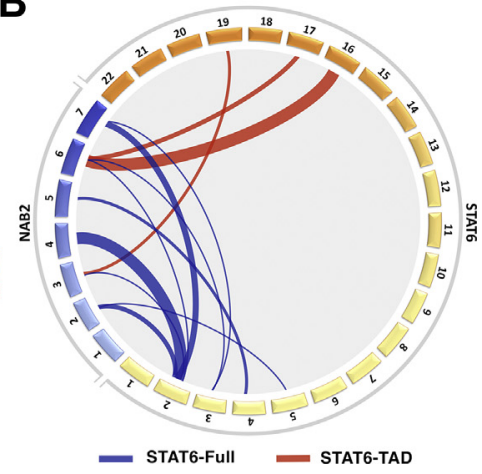

Fig. 1 NAB2-STAT6 fusion variants. (A) Wild-type NAB2 and STAT6 mRNA transcripts with domain structure of their proteins and the most frequent variants of NAB2-STAT6 predicted chimeric proteins. all- $\alpha$, all-alpha domain; CID, CHD4-interacting domain; DBD, DNA-binding domain; NCD1 and NCD2, NAB-conserved domains 1 and 2; PID, protein interaction domain; SH2, Src homology-2 domain; TAD, transactivation domain. (B) Circular plot demonstrating identified fusion variants (shown as links between exons) divided into two groups STAT6-Full (blue) and STAT6-TAD (red).

studies have demonstrated an association between $N A B 2$ ex4-STAT6ex $2 / 3$ and more benign histopathology, but the prognostic value of different fusion variants was not confirmed. ${ }^{3,4,11,12,14,15}$ One potential explanation for the discrepant results could be the shorter follow-up time in the latter studies, given the fact that SFTs commonly recur $>5$ years after initial surgery. ${ }^{16-24}$

In the current study, we aimed to investigate the clinicopathological and prognostic impact of different fusion variants and to explore differences in gene expression in a subset of extrameningeal SFTs selected from our previously reported patient cohort with long-term follow-up. ${ }^{24}$

\section{MATERIALS AND METHODS}

\section{Patient cohort}

This study was performed on a sample of 39 patients selected from our previously published cohort of 100 patients treated with curative intent for localised, extrameningeal SFT. ${ }^{24}$ The cases were collected from our institutional clinical sarcoma database and our pathology database. We selected 19 patients who developed recurrence and 19 patients without recurrence at the time of the last follow-up. One additional patient with tumour recurrence, who was not included in the previous cohort due to neoadjuvant treatment, was also enrolled in the current study. The two groups were paired by anatomical location, year of surgery and method of tissue conservation [fresh frozen or formalin-fixed paraffin-embedded (FFPE) tissue]. The study was approved by the Regional Ethics Committee in South East Norway (\#2010/1244) and conforms to ethical standards in the Declaration of Helsinki. Written informed consent was obtained from all patients.

\section{Histopathological evaluation}

Histopathological parameters were evaluated in the surgical specimens from the primary tumours. Mitotic count was assessed in the most mitotically active areas, counted per 10 high-power fields $\left(1 \mathrm{HPF}=0.2289 \mathrm{~mm}^{2}\right)$ and categorised using a cut-off of $<4$ and $\geq 4$. The presence of necrosis was classified as no necrosis, $<50 \%$ and $\geq 50 \%$. Tumour size was evaluated as a continuous variable. Tumour depth, cellularity, atypia/pleomorphism, infiltrative growth pattern, resection margins and the prognostic scoring system G-score were classified and subgrouped as reported in the former study. ${ }^{24}$ Immunohistochemistry was performed as reported previously. ${ }^{24}$

\section{Extraction and quantification of RNA}

Fresh frozen or FFPE tumour samples from primary tumour resection specimens were collected for all cases. Tumour content of the specimen was above $90 \%$ in all samples with minimal amount of necrosis or normal tissue. RNA from FFPE samples was isolated by truXTRACT FFPE microTUBE RNA Kit (Covaris, USA) according to the manufacturer's instructions. Two to three 20 $\mu \mathrm{m}$ sections were used. RNA from fresh frozen samples was isolated by miRNeasy Mini Kit (Qiagen, USA) according to the manufacturer's instructions, using 30-50 mg of tumour tissue. Extracted RNA was stored at $-80^{\circ} \mathrm{C}$. Concentration and purity of RNA were analysed by NanoDrop 2000 spectrophotometer (Thermo Fisher Scientific, USA). For all cases, 280/260 and $260 / 230$ ratios $>1.8$ were achieved. RNA integrity was evaluated by Agilent 2100 Bioanalyzer (Agilent Technologies, USA) and an acceptable level of DV200 $\geq 30 \%$ was achieved in all but three cases, which were withdrawn from further analysis.

\section{Library construction and next generation sequencing}

Libraries were made by the Oslo University Hospital Genomics Core Facility (www.oslo.genomics.no) using the TruSight RNA Pan-Cancer Panel (Illumina, USA) according to the manufacturer's instructions. One hundred nanograms of total RNA was used as input material for library preparation. Two samples were not sequenced due to unsuccessful library preparation. The resulting libraries of the 34 final samples were sequenced paired-end on a NextSeq500 instrument (Illumina).

\section{Analysis of fusion data}

FASTQ files were further processed by the RNA-Seq Alignment pipeline, version 1.1.0 (Illumina). Reads were mapped to human (UCSC hg19) reference genome using STAR aligner (version STAR_2.5.0b). ${ }^{25}$ Fusion detection was performed using the Manta algorithm ${ }^{26}$ and STAR aligner, and gene counts per transcript were estimated using Cufflinks 2 (http://cole-trapnelllab.github.io/cufflinks/). Detected fusions were further inspected manually by uploading alignment BAM files into the Integrative Genomics Viewer (IGV), version 2.4.14. ${ }^{27}$ NM_005967 transcript variant for NAB2 and NM_001178078 for STAT6 were chosen to call the fusions.

\section{Gene expression analysis}

The gene count was normalised using 'regularised log' (rlog) function of DESeq2. ${ }^{28}$ Principal component analysis (PCA) and heatmap analysis were performed on the rlog normalised counts data. The differential expression analysis was performed using DESeq2 and the genes with BenjaminiHochberg adjusted $p$ values (padj) $\leq 0.05$ and $\log 2$ FoldChange (LFC) $\geq 1$ were considered as differentially expressed.

\section{Statistical analysis}

Recurrence-free interval (RFi) and distant recurrence-free interval (D-RFi) ${ }^{29}$ were calculated from the date of diagnosis until date of distant metastasis or local recurrence for RFi and distant metastasis for D-RFi. Patients without an event were censored at date of last radiological examination of chest and/or abdomen. Death was not considered an event. For overall survival (OS) date of death of any cause was collected from the National Registry of Norway and patients still alive were censored at 21 February 2019. Associations between fusion groups and clinicopathological parameters were examined by means of two-tailed Fisher's exact test for categorical variables and Mann-Whitney U 
test for continuous variables, as appropriate. Kaplan-Meier analysis was performed to estimate RFi, D-RFi and OS and log-rank test was used to compare groups. A $p$ value $<0.05$ was considered significant. SPSS Statistics, version 25.0 for Mac (SPSS, USA) was used.

\section{RESULTS}

\section{Patient cohort and histopathological characteristics}

Thirty-nine patients with localised, extrameningeal SFT were included in this study, of whom 20 developed distant recurrence and 19 were without recurrence after long term followup (median 97 months, range 0-220 months). Demographic and clinicopathological characteristics are presented in Table 1 . There were 24 females and 15 males with median age at diagnosis of 62 years (range 37-82). The most frequent tumour location was abdomen/retroperitoneum (46\%), followed by extremity (23\%) and pleura/lung (18\%). Median tumour size was $11 \mathrm{~cm}$ (range $2-28 \mathrm{~cm}$ ). Two patients $(5 \%)$ underwent adjuvant radiation therapy and one patient received neoadjuvant chemotherapy $(3 \%)$. There were 14 tumours $(36 \%)$ with $\geq 4$ mitotic figures per $10 \mathrm{HPF}$. Necrosis was present in 17 tumours $(44 \%)$ including three tumours $(8 \%)$ with $\geq 50 \%$ necrosis. All cases showed strong nuclear STAT6 immunostaining.

Table 1 Demographic, clinical and histopathological characteristics

\begin{tabular}{|c|c|}
\hline Characteristics & $\begin{array}{c}\text { Patients }(\mathrm{N}=39) \\
n(\%)^{\mathrm{a}}\end{array}$ \\
\hline \multicolumn{2}{|l|}{ Age (at presentation), years } \\
\hline Median (range) & $62(37-82)$ \\
\hline \multicolumn{2}{|l|}{ Gender } \\
\hline Male & $15(38)$ \\
\hline Female & $24(62)$ \\
\hline \multicolumn{2}{|l|}{ Site } \\
\hline Head and neck & $4(10)$ \\
\hline Extremity & $9(23)$ \\
\hline Trunk wall & $1(3)$ \\
\hline Pleura/lung & $7(18)$ \\
\hline Abdomen/retroperitoneum & $18(46)$ \\
\hline \multicolumn{2}{|l|}{ Tumour depth } \\
\hline Deep-seated & $35(90)$ \\
\hline Superficial & $4(10)$ \\
\hline \multicolumn{2}{|l|}{ Tumour size, cm } \\
\hline Median (range) & $11(2-28)$ \\
\hline \multicolumn{2}{|l|}{ Resection margins } \\
\hline R0 & $24(62)$ \\
\hline $\mathrm{R} 1$ & $13(33)$ \\
\hline $\mathrm{R} 2$ & $2(5)$ \\
\hline \multicolumn{2}{|l|}{ Mitotic count ${ }^{\mathrm{b}}$} \\
\hline Median (range) & $2(0-50)$ \\
\hline$<4$ & $25(64)$ \\
\hline$\geq 4$ & $14(36)$ \\
\hline \multicolumn{2}{|l|}{ Necrosis } \\
\hline Absent & $22(56)$ \\
\hline$<50 \%$ & $14(36)$ \\
\hline$\geq 50 \%$ & $3(8)$ \\
\hline \multicolumn{2}{|l|}{ Atypia/pleomorphism } \\
\hline No & $32(82)$ \\
\hline Yes & $7(18)$ \\
\hline \multicolumn{2}{|l|}{ Cellularity } \\
\hline Low & $1(3)$ \\
\hline Moderate & $16(41)$ \\
\hline High & $22(56)$ \\
\hline \multicolumn{2}{|l|}{ Growth pattern } \\
\hline Pushing & $24(62)$ \\
\hline Infiltrating & $15(38)$ \\
\hline
\end{tabular}

${ }^{\text {a }}$ Unless otherwise specified.

${ }^{\mathrm{b}}$ Number of mitotic figures per 10 high-power fields.

\section{Capture-based RNA sequencing}

To investigate NAB2-STAT6 fusion variants and gene expression profiles, capture-based RNA sequencing using the Illumina TruSight RNA Pan-Cancer Panel was performed. In five cases RNA extraction or library preparation was unsuccessful. NAB2-STAT6 fusions were detected in all the 34 sequenced cases. In total, 12 fusion variants were identified (Fig. 1B; Supplementary Table 1, Appendix A). The most frequent variants were NAB2ex6-STAT6ex16 (8 cases, 23\%) and NAB2ex4-STAT6ex2 (7 cases, 21\%) (Fig. 1A). We divided the fusion gene variants into two groups according to which STAT6 domains were present in the predicted fusion protein. Thirteen tumours $(38 \%)$ were categorised into the STAT6-TAD group, which included cases with only the TAD domain of STAT6 present in the fusion protein. Twenty-one tumours (62\%) were categorised into the STAT6-Full group, including cases with almost full length STAT6 involved in the fusion (Fig. 1B).

To identify differences in the transcriptional signature between STAT6-TAD and STAT6-Full tumours, expression analysis of the 1385 genes from the Pan-Cancer Panel was performed. In principal component analysis, three distinct outliers were observed, presumably as a result of an increased number of mast cells in one case (SFT15) and technical reasons in two other cases (SFT14 and SFT17; Supplementary Fig. 1A, Appendix A). After removal of outliers, a substantial difference between fresh frozen and FFPE samples was evident, with a larger variance in gene expression for FFPE samples (Supplementary Fig. 1B, Appendix A). To avoid the risk that fixation methods may influence the gene expression data, all FFPE samples were excluded from further analysis. Thus, 20 fresh frozen samples remained for differential expression analysis. Twenty-six differentially expressed genes were identified, comparing the STAT6-TAD and STAT6-Full groups (Fig. 2A). STAT6TAD samples had higher expression of several genes including BAIAP2L1, CEBPA and BIN1. The STAT6-Full group showed notable gene expression diversity between the samples. STAT6 had higher expression in all STAT6-Full samples. Moreover, IGF2, SPARC, HSPG2, FBLN1 and COL14A1 were highly expressed in a subset of the STAT6Full group.

Next, differential gene expression analysis was performed comparing NAB2-STAT6 fusion variants in tumours from patients who developed recurrence (Rec) and from patients without recurrence (NoRec). In the NoRec group two main clusters were observed with a higher expression of four genes, including $F G F 2$, in the STAT6-TAD group and eight genes, including $P D G F R B$, in the STAT6-Full group (Fig. 2B). A total of 46 differentially expressed genes were found in the Rec group, where the STAT6-Full cluster, having three samples in total, was dominated by two samples with high expression of EGR2, IGF2, STAT6 and genes involved in production of extracellular matrix (SPARC, COL12A1, COL14A1, FBLN1, HSPG2, FN1) (Fig. 2C). No clear association between the clusters and anatomical location of the tumours was observed (data not shown).

\section{Association between fusion variants and clinicopathological characteristics}

Associations between fusion variants and clinicopathological characteristics are summarised in Table 2. All the 

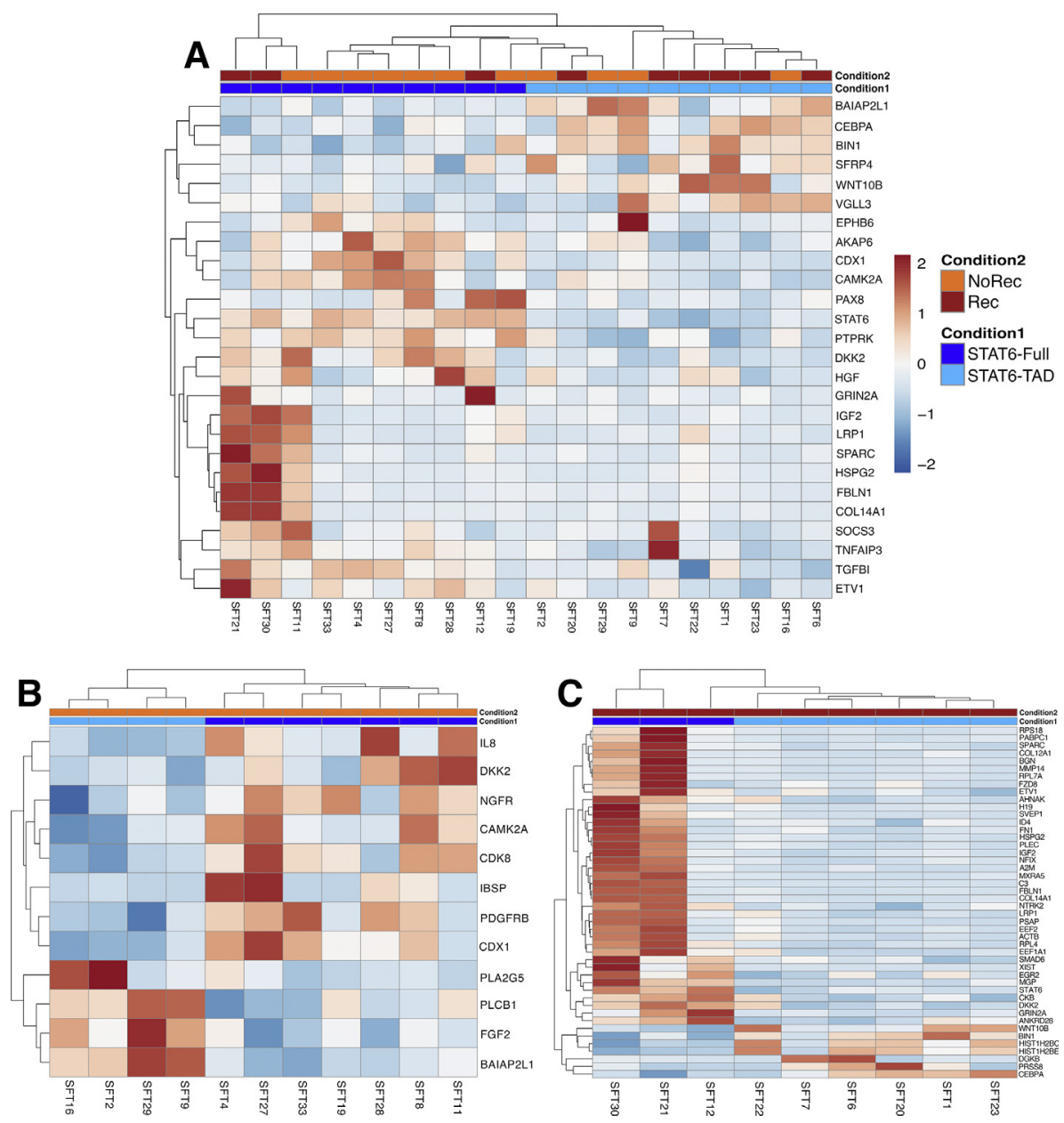

Fig. 2 Differential gene expression analysis. (A) Heat map comparison of differentially expressed genes between STAT6-TAD and STAT6-Full fusion groups, (B) for tumours without recurrence (NoRec) and (C) for tumours with distant recurrence (Rec).

pleuropulmonary tumours had the NAB2ex4-STAT6ex2 fusion variant, whereas tumours in other locations expressed fusion variants belonging to both groups. Tumours in the STAT6-TAD group had significantly higher mitotic count compared to STAT6-Full tumours (median 4 vs 1 per $10 \mathrm{HPF}, p=0.016)$. According to our recently identified risk stratification model (G-score), ${ }^{24}$ STAT6TAD tumours were scored as high risk tumours in $62 \%$ of the cases compared to $19 \%$ in the STAT6-Full group $(p=0.037)$. No statistically significant associations between fusion groups and age, gender, tumour size, resection margin, tumour depth, necrosis, cellularity, atypia and growth pattern were observed. However, patients in the STAT6-Full group were older (median 62 vs 54 years) and had slightly larger tumours (median 13 vs $11 \mathrm{~cm}$ ) with less necrosis (33\% vs $62 \%)$.

\section{Patient outcome and fusion variants}

Median follow-up for OS for the patients still alive was 127 months (range 40-308 months) and median follow-up for $\mathrm{RFi}$ for patients without recurrence was 97 months (range 0-220 months). Median time to recurrence was 61 months (range 3-227). All twenty patients with recurrence were selected for this study because they had developed distant recurrence. Seven of these patients (35\%) also developed local recurrence, of whom four had their local recurrence as the first event. Lung (45\%) and abdomen (35\%) were the most frequent first metastatic sites. Locally recurrent tumours were retroperitoneal in five cases and pleuropulmonary in two cases. Nineteen patients died during follow-up, of whom $11(57 \%)$ died of SFT, two (11\%) for other reasons and six (32\%) of unknown cause.

Of 15 patients with distant recurrence and known fusion status, seven belonged to the STAT6-Full group and eight to the STAT6-TAD group. Four patients with STAT6-Full tumours and two patients with STAT6-TAD tumours experienced local recurrence. Median time to recurrence for STAT6-TAD tumours was 27 months compared to 65 months in the STAT6-Full group. Patients in the STAT6TAD group had shorter RFi (Fig. 3A, $p=0.004$ ), D-RFi (Fig. 3B, $p=0.006$ ) and OS (Fig. 3C, $p=0.012$ ). Estimated 10year RFi rate for patients with STAT6-TAD tumours was $25 \%$ compared to $78 \%$ for the STAT6-Full group.

\section{DISCUSSION}

In the present study, we have investigated the clinical impact of different NAB2-STAT6 fusion variants and explored gene expression patterns in extrameningeal SFT. Two fusion 
Table 2 Associations between fusion variants and clinicopathological characteristics

\begin{tabular}{|c|c|c|c|}
\hline \multirow[t]{2}{*}{ Characteristics } & \multicolumn{2}{|c|}{ Patients, $n(\%)^{\mathrm{a}}$} & \multirow[t]{2}{*}{$p$ value } \\
\hline & STAT6-Full & STAT6-TAD & \\
\hline Number of patients $(\mathrm{N}=34)$ & 21 & 13 & \\
\hline Age, years, median & 62 & 54 & 0.993 \\
\hline Tumour size, $\mathrm{cm}$, median & 13 & 11 & 0.841 \\
\hline Mitotic count per $10 \mathrm{HPF}$, median & 1 & 4 & 0.016 \\
\hline Sex & & & 0.728 \\
\hline Female & $10(67)$ & $5(33)$ & \\
\hline Male & $11(58)$ & $8(42)$ & \\
\hline Location $^{\mathrm{b}}$ & & & 0.021 \\
\hline Extremity / trunk wall / head and neck & $4(19)$ & $8(62)$ & \\
\hline Pleura/lung & $5(24)$ & $0(0)$ & \\
\hline Abdomen/retroperitoneum & $12(57)$ & $5(38)$ & \\
\hline Necrosis ${ }^{\mathrm{b}}$ & & & 0.160 \\
\hline No & $14(67)$ & $5(38)$ & \\
\hline$<50 \% / \geq 50 \%$ & $7(33)$ & $8(62)$ & \\
\hline High & $9(43)$ & $9(69)$ & \\
\hline Atypia/pleomorphism & & & 0.653 \\
\hline Yes & $3(14)$ & $3(21)$ & \\
\hline No & $18(86)$ & $11(79)$ & \\
\hline Tumour depth & & & 0.279 \\
\hline Deep & $20(95)$ & $11(79)$ & \\
\hline Superficial & $1(5)$ & $3(21)$ & \\
\hline Infiltrative growth pattern & & & 1.000 \\
\hline Pushing & $13(62)$ & $8(57)$ & \\
\hline Infiltrating & $8(38)$ & $6(43)$ & \\
\hline G-score & & & $\mathbf{0 . 0 3 7}$ \\
\hline Low risk & $6(29)$ & $3(23)$ & \\
\hline Intermediate risk & $11(52)$ & $2(15)$ & \\
\hline High risk & $4(19)$ & $8(62)$ & \\
\hline
\end{tabular}

HPF, high-power field.

Significant $p$ values are in bold

${ }^{a}$ Unless otherwise specified.

${ }^{\mathrm{b}}$ Extremity / trunk wall / head and neck, low and moderate cellularity, necrosis $<50 \%$ and $\geq 50 \%$ were grouped for analysis.

groups were defined based on the length of the STAT6 gene, STAT6-Full and STAT6-TAD. Patients with STAT6-TAD fusions had a higher mitotic count and an increased risk of recurrence compared to STAT6-Full tumours, and distinct transcriptional signatures for each fusion group were identified.
Patients in the STAT6-TAD group had inferior RFi, D-RFi and OS, and median time to recurrence was less than half compared to the STAT6-Full group (27 vs 65 months). These results are consistent with and complement the observations made by Barthelmess and co-workers, ${ }^{13}$ who were the first to show that STAT6-TAD tumours more often recur. Several

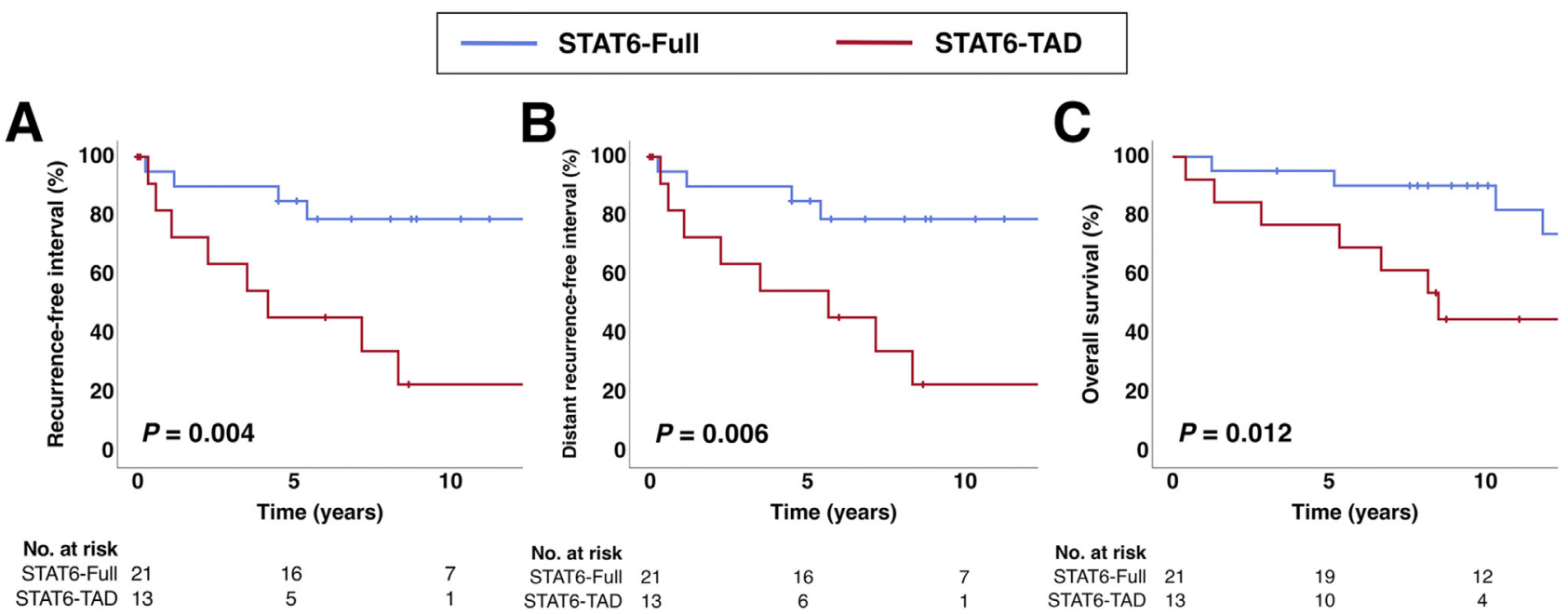

Fig. 3 Associations between fusion groups and outcome. Kaplan-Meier survival curves of (A) recurrence-free interval, (B) distant recurrence-free interval, and (C) overall survival, stratified based on fusion groups. 
later studies have not been able to confirm the prognostic impact of the fusion variants. ${ }^{3,4,11,12}$ However, this discrepancy could be explained by short follow-up time with few detected recurrences, $3,4,11,12$ and heterogeneous inclusion criteria, such as inclusion of meningeal SFTs ${ }^{3,11}$ and series with only intrathoracic cases. ${ }^{4}$ Our cohort with long term follow-up and only extrameningeal tumours clearly shows that STAT6-TAD tumours have an inferior prognosis, but whether the fusion variant has independent prognostic impact is yet to be investigated. Pleuropulmonary tumours frequently contain a STAT6-Full fusion variant and have a favourable prognosis, and anatomical location might be a confounder.

STAT6-TAD tumours showed a significantly higher mitotic count and higher G-score (recurrence risk score) compared to the STAT6-Full group, and had smaller tumour size and more necrosis, albeit not statistically significant. The findings are consistent with previously reported data, and reinforce the presumption by Barthelmess et al. that the tumours with NAB2ex6-STAT6ex 16/17 fusions exhibit a more malignant phenotype and clinical behaviour. ${ }^{3,11,13}$

The breakpoints in STAT6 seem to cluster only at the $3^{\prime}$ or $5^{\prime}$ end of the gene, in contrast to $N A B 2$ that displays a wide distribution of breakpoints throughout the length of the gene. This observation was the basis for our definition of the fusion groups. Most of the predicted NAB2-STAT6 fusion proteins have a truncated NAB2 repression domain and a preserved STAT6 TAD. The STAT6-Full fusion variants also contain an intact all-alpha domain, DBD and SH2 domain and an intact or truncated PID of STAT6. The observed differences between the two fusion variant groups are likely related to the absence of one or several of these four STAT6 domains. For example, it might alter the DNA binding specificity of the fusion protein or modify the recruitment of coactivators thereby transactivating activity. To precisely elucidate the molecular basis for the different biological functions, isogenic model systems expressing the different fusion variants would be needed.

Differential gene expression analysis of the STAT6-TAD and STAT6-Full groups revealed 26 significantly differently expressed genes. To avoid the potential confounding effect of biological differences between the fusion variants, the analysis was also performed separately in tumours with and without recurrence. One of the transcriptional programs identified was the EGR1 pathway, which is known to be activated in SFT, ${ }^{1}$ and included the EGR1-responsive genes $F G F 2, I G F 2$ and $E G R 2$. Fibroblast growth factor 2 (FGF2), which is directly upregulated by EGR $1,{ }^{30}$ was more highly expressed in STAT6-TAD tumours, consistent with its role as an activator of cell proliferation. ${ }^{31,32}$ IGF2 and EGR2 had increased expression in the STAT6-Full group, suggesting differential regulation of EGR1 pathways between the fusion groups. Increased expression of IGF2 in SFTs compared to other mesenchymal tumours is well known. ${ }^{32,33}$ IGF2 has mitogenic and anti-apoptotic potential and is linked to refractory hypoglycaemia in SFT patients. ${ }^{34}$ None of the patients in our cohort showed signs of paraneoplastic hypoglycaemia, which is in line with the observation that expression levels of $I G F 2$ do not always correlate with clinical evidence of hypoglycaemia. ${ }^{35}$ Higher expression of $E G R 2$, stimulating fibrogenesis, ${ }^{36}$ and other genes involved in the production of extracellular matrix (SPARC, COL12A1, COL14A1, FBLN1, HSPG2, FN1) were observed in STAT6Full tumours. This is consistent with the previously reported association between more prominent diffuse fibrous stroma and NAB2ex4-STAT6ex2/3 fusion. ${ }^{13}$ Interestingly, a prominent fibrous stroma was observed in 10 of 21 (48\%) tumours with STAT6-Full fusions, compared to three of $13(23 \%)$ in the STAT6-TAD group (data not shown). STAT6 was more highly expressed in the STAT6-Full group. We believe that the difference in the number of STAT6 exons and, hence, in the number of STAT6 RNA transcripts between the fusion groups might have contributed to the observed differential expression of the gene. Based on current knowledge, STAT6 is mostly involved in allergic and immune signalling pathways, but also appears to have a role in oncogenesis. ${ }^{37,38}$

Several tyrosine kinase inhibitors have antitumour activity in SFT, including sunitinib and pazopanib. ${ }^{39-41}$ However, the clinical and radiological response varies considerably between patients, suggesting that different biological mechanisms might be activated in different tumours. Sunitinib's antitumour activity in SFT is mediated, at least partly, through a platelet derived growth factor receptor beta (PDGFR $\beta$ )-dependent mechanism. ${ }^{39}$ PDGFRB expression was higher in STAT6-Full tumours, suggesting that sunitinib might show increased efficacy in this group. The multi-kinase inhibitor pazopanib, also active against PDGFR $\beta$, has recently shown positive results in clinical trials in SFT, ${ }^{40,41}$ and it would be interesting to correlate fusion gene status with response in these trials. Given high expression of $I G F 2$, targeting of IGF pathways could be a promising strategy for the STAT6-Full group. In contrast, STAT6-TAD patients could benefit from anti-FGFR targeted therapy, based on the increased expression of FGF2. Certainly, this remains speculative and must be investigated further in preclinical and clinical studies.

Our study has certain limitations. The small sample size with relatively few cases in each group restricted the possibility for multivariable survival analysis, which would be needed to investigate whether the fusion variants contribute additional prognostic information beyond standard clinicopathological factors. The performance of the differential gene expression analysis was also limited by the small number of samples and the heterogeneity within the groups. Moreover, all pleuropulmonary tumours in our cohort harboured the NAB2ex4-STAT6ex2 fusion and a confounding effect of anatomical localisation cannot be excluded. Finally, we used a preselected panel of cancer related genes, and the number of included genes is insufficient to perform a meaningful pathway enrichment analysis. A study utilising whole transcriptome sequencing of a higher number of fresh frozen samples would thus be advantageous.

In conclusion, we have shown that NAB2-STAT6 fusion variants in SFT can be divided into two groups, STAT6-TAD and STAT6-Full, with distinct clinicopathological and molecular profiles. Patients with STAT6-TAD tumours have an increased risk of recurrence, but whether fusion status has independent prognostic impact is not clarified. The prognostic value of fusion variants should be further explored in larger patient cohorts, emphasising the need for continued international collaboration in this rare disease. 
Conflicts of interest and sources of funding: The work was supported by Norwegian Cancer Society (grants 1443852014, 418589-2013 and 198034-2019) and the South-East Norway Regional Health Authority (grants 2019064, 2019011). The authors state that there are no conflicts of interest to disclose.

\section{APPENDIX A. SUPPLEMENTARY DATA}

Supplementary data to this article can be found online at https://doi.org/10.1016/j.pathol.2020.11.010.

Address for correspondence: Dr Kjetil Boye, Department of Oncology, Norwegian Radium Hospital, Oslo University Hospital, Box 4953 Nydalen, NO-0424, Oslo, Norway. E-mail: kjetil.boye@rr-research.no

\section{References}

1. Robinson DR, Wu YM, Kalyana-Sundaram S, et al. Identification of recurrent NAB2-STAT6 gene fusions in solitary fibrous tumor by integrative sequencing. Nat Genet 2013; 45: 180-5

2. Chmielecki J, Crago AM, Rosenberg M, et al. Whole-exome sequencing identifies a recurrent NAB2-STAT6 fusion in solitary fibrous tumors. Nat Genet 2013; 45: 131-2.

3. Akaike K, Kurisaki-Arakawa A, Hara K, et al. Distinct clinicopathological features of NAB2-STAT6 fusion gene variants in solitary fibrous tumor with emphasis on the acquisition of highly malignant potential. Hum Pathol 2015; 46: 347-56.

4. Huang SC, Li CF, Kao YC, et al. The clinicopathological significance of NAB2-STAT6 gene fusions in 52 cases of intrathoracic solitary fibrous tumors. Cancer Med 2016; 5: 159-68.

5. Doyle LA, Vivero M, Fletcher CD, et al. Nuclear expression of STAT6 distinguishes solitary fibrous tumor from histologic mimics. Mod Pathol 2014; 27: 390-5.

6. Demicco EG, Harms PW, Patel RM, et al. Extensive survey of STAT6 expression in a large series of mesenchymal tumors. Am J Clin Pathol 2015; 143: 672-82.

7. Yoshida A, Tsuta K, Ohno M, et al. STAT6 immunohistochemistry is helpful in the diagnosis of solitary fibrous tumors. Am J Surg Pathol 2014; 38: 552-9.

8. Srinivasan R, Mager GM, Ward RM, et al. NAB2 represses transcription by interacting with the CHD4 subunit of the nucleosome remodeling and deacetylase (NuRD) complex. J Biol Chem 2006; 281: $15129-37$.

9. Mitchell AL, Attwood TK, Babbitt PC, et al. InterPro in 2019: improving coverage, classification and access to protein sequence annotations. Nucleic Acids Res 2019; 47: D351-60.

10. Nakada S, Minato H, Nojima T. Clinicopathological differences between variants of the NAB2-STAT6 fusion gene in solitary fibrous tumors of the meninges and extra-central nervous system. Brain Tumor Pathol 2016; 33: 169-74.

11. Tai HC, Chuang IC, Chen TC, et al. NAB2-STAT6 fusion types accoun for clinicopathological variations in solitary fibrous tumors. Mod Pathol 2015; 28: 1324-35.

12. Chuang IC, Liao KC, Huang HY, et al. NAB2-STAT6 gene fusion and STAT6 immunoexpression in extrathoracic solitary fibrous tumors: the association between fusion variants and locations. Pathol Int 2016; 66: 288-96.

13. Barthelmess S, Geddert $\mathrm{H}$, Boltze $\mathrm{C}$, et al. Solitary fibrous tumors/ hemangiopericytomas with different variants of the NAB2-STAT6 gene fusion are characterized by specific histomorphology and distinct clini copathological features. Am J Pathol 2014; 184: 1209-18.

14. Yuzawa S, Nishihara H, Wang L, et al. Analysis of NAB2-STAT6 gene fusion in 17 cases of meningeal solitary fibrous tumor/hemangioper icytoma: review of the literature. Am J Surg Pathol 2016; 40: 1031-40.

15. Park HK, Yu DB, Sung M, et al. Molecular changes in solitary fibrous tumor progression. J Mol Med (Berl) 2019; 97: 1413-25.

16. Tapias LF, Mino-Kenudson M, Lee H, et al. Risk factor analysis for the recurrence of resected solitary fibrous tumours of the pleura: a 33-year experience and proposal for a scoring system. Eur $J$ Cardiothorac Surg 2013; 44: 111-7.

17. Salas S, Resseguier N, Blay JY, et al. Prediction of local and metastatic recurrence in solitary fibrous tumor: construction of a risk calculator in a multicenter cohort from the French Sarcoma Group (FSG) database. Ann Oncol 2017; 28: 1979-87.

18. Reisenauer JS, Mneimneh W, Jenkins S, et al. Comparison of risk stratification models to predict recurrence and survival in pleuropulmonary solitary fibrous tumor. J Thorac Oncol 2018; 13: 1349-62.

19. Gholami S, Cassidy MR, Kirane A, et al. Size and location are the most important risk factors for malignant behavior in resected solitary fibrous tumors. Ann Surg Oncol 2017; 24: 3865-71.

20. Vaz Salgado MA, Soto M, Reguero ME, et al. Clinical behavior of solitary fibrous tumor: a retrospective review of 30 patients. Clin Transl Oncol 2017; 19: 357-63.

21. Demicco EG, Park MS, Araujo DM, et al. Solitary fibrous tumor: a clinicopathological study of 110 cases and proposed risk assessment model. Mod Pathol 2012; 25: 1298-306.

22. Demicco EG, Griffin AM, Gladdy RA, et al. Comparison of published risk models for prediction of outcome in patients with extrameningeal solitary fibrous tumour. Histopathology 2019; 75: 723-37.

23. Baldi GG, Stacchiotti S, Mauro V, et al. Solitary fibrous tumor of all sites: outcome of late recurrences in 14 patients. Clin Sarcoma Res 2013; 3: 4 .

24. Georgiesh T, Boye K, Bjerkehagen B. A novel risk score to predict early and late recurrence in solitary fibrous tumour. Histopathology 2020; 77 $123-32$

25. Dobin A, Davis CA, Schlesinger F, et al. STAR: ultrafast universal RNA-seq aligner. Bioinformatics 2013; 29: 15-21.

26. Chen X, Schulz-Trieglaff O, Shaw R, et al. Manta: rapid detection of structural variants and indels for germline and cancer sequencing applications. Bioinformatics 2016; 32: 1220-2.

27. Robinson JT, Thorvaldsdottir H, Winckler W, et al. Integrative genomics viewer. Nat Biotechnol 2011; 29: 24-6.

28. Love MI, Huber W, Anders S. Moderated estimation of fold change and dispersion for RNA-seq data with DESeq2. Genome Biol 2014 15: 550 .

29. Gourgou-Bourgade S, Cameron D, Poortmans P, et al. Guidelines for time-to-event end point definitions in breast cancer trials: results of the DATECAN initiative (Definition for the Assessment of Time-to-event Endpoints in CANcer trials). Ann Oncol 2015; 26: 873-9.

30. Wang D, Mayo MW, Baldwin Jr AS. Basic fibroblast growth factor transcriptional autoregulation requires EGR-1. Oncogene 1997; 14 2291-9.

31. Kottakis F, Polytarchou C, Foltopoulou P, et al. FGF-2 regulates cell proliferation, migration, and angiogenesis through an NDY1/KDM2BmiR-101-EZH2 pathway. Mol Cell 2011; 43: 285-98.

32. Hajdu M, Singer S, Maki RG, et al. IGF2 over-expression in solitary fibrous tumours is independent of anatomical location and is related to loss of imprinting. J Pathol 2010; 221: 300-7.

33. Steigen SE, Schaeffer DF, West RB, et al. Expression of insulin-like growth factor 2 in mesenchymal neoplasms. Mod Pathol 2009; 22 914-21.

34. de Groot JW, Rikhof B, van Doorn J, et al. Non-islet cell tumourinduced hypoglycaemia: a review of the literature including two new cases. Endocr Relat Cancer 2007; 14: 979-93.

35. Han G, Zhang Z, Shen X, et al. Doege-Potter syndrome: a review of the literature including a new case report. Medicine (Baltimore) 2017; 96 : e7417.

36. Fang F, Ooka K, Bhattacharyya S, et al. The early growth response gene Egr2 (Alias Krox20) is a novel transcriptional target of transforming growth factor-beta that is up-regulated in systemic sclerosis and mediates profibrotic responses. Am J Pathol 2011; 178: 2077-90.

37. Shahmarvand N, Nagy A, Shahryari J, et al. Mutations in the signal transducer and activator of transcription family of genes in cancer. Cancer Sci 2018; 109: 926-33.

38. Binnemars-Postma K, Bansal R, Storm G, et al. Targeting the Stat6 pathway in tumor-associated macrophages reduces tumor growth and metastatic niche formation in breast cancer. FASEB J 2018; 32: $969-78$.

39. Stacchiotti S, Negri T, Palassini E, et al. Sunitinib malate and figitumumab in solitary fibrous tumor: patterns and molecular bases of tumo response. Mol Cancer Ther 2010: 9: 1286-97.

40. Martin-Broto J, Stacchiotti S, Lopez-Pousa A, et al. Pazopanib for treatment of advanced malignant and dedifferentiated solitary fibrous tumour: a multicentre, single-arm, phase 2 trial. Lancet Oncol 2019; 20: $134-44$

41. Martin-Broto J, Cruz J, Penel N, et al. Pazopanib for treatment of typical solitary fibrous tumours: a multicentre, single-arm, phase 2 trial. Lancet Oncol 2020; 21: 456-66. 